\title{
Growth Mechanisms of Electron Beam Induced Carbon Deposition Using Hydrocarbon Contamination
}

\author{
A.D. Garetto*, D.P. Griffis*, P.E. Russell*, J. Fowlkes**, P.D. Rack** \\ * Materials Science and Engineering Department and Analytical Instrumentation Facility, \\ North Carolina State University, 2410 Campus Shore Drive, 318 EGRC, Raleigh, NC \\ 27695 \\ ** Dept. of Materials Science and Engineering, 434 Dougherty Hall, The University of \\ Tennessee, Knoxville, TN 37996-2200
}

Electron beam induced micromachining employs an electron beam to irradiate precursor molecules adsorbed on a substrate in order to induce a chemical reaction that results in removal of substrate material or in deposition of non-volatile materials. This technique offers many promising applications in the field of nanofabrication and has a major benefit over focused ion beam machining in that there is no ion implantation.

This work investigates the mechanisms which limit growth of carbon nano-structures in the scanning electron microscope. While it is known that these structures result from hydrocarbon contamination, the source of the hydrocarbons is unknown and could result from numerous sources including back streaming pump oil, exposure to air, etc. Possible mechanisms affecting the supply of hydrocarbons to the electron beam point of impact (focused, non rastered beam) include surface diffusion, vapor phase supply, or a combination of the two. Experiments were designed to limit possible surface diffusion of adsorbed hydrocarbons on the sample surface in order to determine if this is the dominant mechanism.

All growth and subsequent imaging will be performed using a JEOL 6400FE field emission SEM and all structures were grown on Au coated Si. The structures shown in Figure 1 illustrate the versatility of this growth technique. Figure 2 shows the results obtained using line scans to laterally grow several free standing rods and then cross these rods perpendicularly. The horizontal growth across and perpendicular to the free standing vertical growths having different separations illustrates the limiting effect of surface diffusion i.e. growth of the horizontal rod between the two most widely separated vertical rods is not continuous due to lack of precursor supply.

In order to further test the surface diffusion hypothesis, Focused Ion Beam (FIB) micromachining utilizing an FEI FIB 610 with a beam patterning program will be used to micromachine circular trenches into the Au coated Si sample producing a Si pillar (figure 3 ). These samples will then be plasma cleaned to remove hydrocarbon contamination and electron beam induced hydrocarbon structures will be grown on top of the micromachined central pillar. The high aspect ratio trench surrounding the pillar should restrict the diffusion of any vapor phase hydrocarbon precursors present in the SEM chamber from adsorbing onto the sample thus restricting electron beam induced growth. Initial (Figure 4) and new results of subsequent experiments will be presented. 
In collaboration with researchers at the University of Tennessee, a simulation of the electron-beam induced deposition process (coded using the Matlab ${ }^{\circledR}$ program) is being utilized to model these results.

References:

1) Mooney, C.B. (1995). Unpublished Masters’ Thesis. North Carolina State University.

2) Matsui, Shinji. Proceedings of the IEEE, Vol.85, NO.4 (1997) 629-643.

3) Fujita, J. et al., J. Vac. Sci. Technol. B 21(6), (Nov/Dec 2003) 2990.
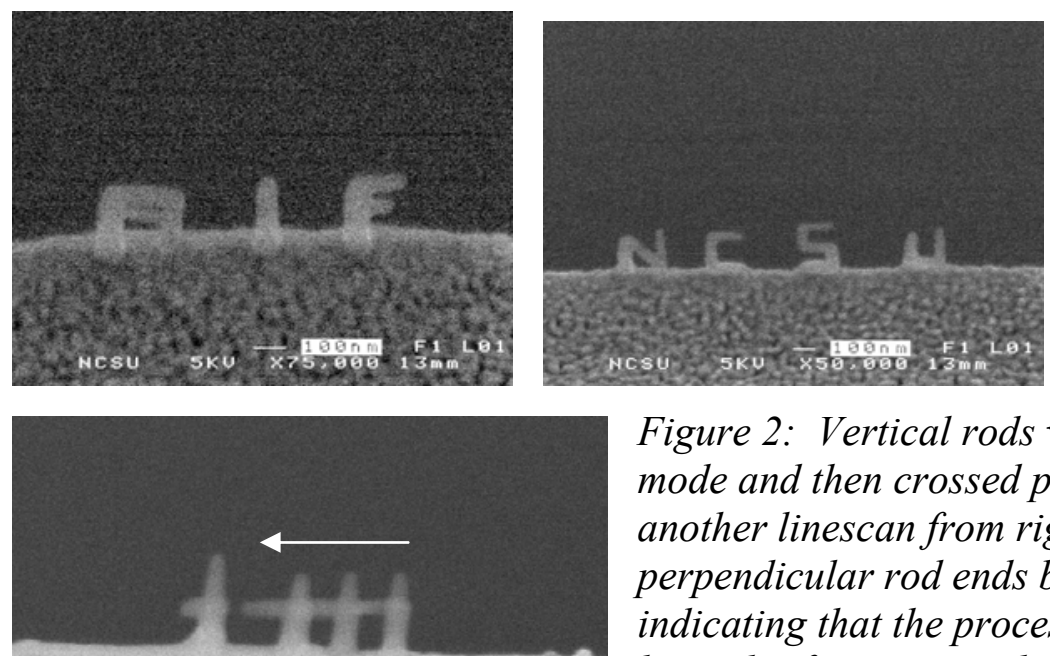

Figure 1: Acronyms for AIF and NCSU grown from hydrocarbon contamination in a JEOL FE-6400. Beam conditions were $5 \mathrm{kV}, 2.6$ pA, 1E-6 Torr for both growth and imaging

Figure 2: Vertical rods were grown using linescan mode and then crossed perpendicularly with another linescan from right to left. The perpendicular rod ends before reaching final pillar indicating that the process is surface diffusion limited. If it was not, the rod would grow all the way because it is still being supplied by the vapor phase.

Figure 3: FIB milled trench with a pillar in the center. With a high aspect ratio trench, a very low concentration of hydrocarbons from vapor phase will adsorb to the bottom and sides. Growth is performed off the pillar using up hydrocarbons adsorbed to the pillar and thus limiting the surface diffused supply from the substrate surface. Hydrocarbons must diffuse all the way down the trench and back up the pillar to contribute to growth.

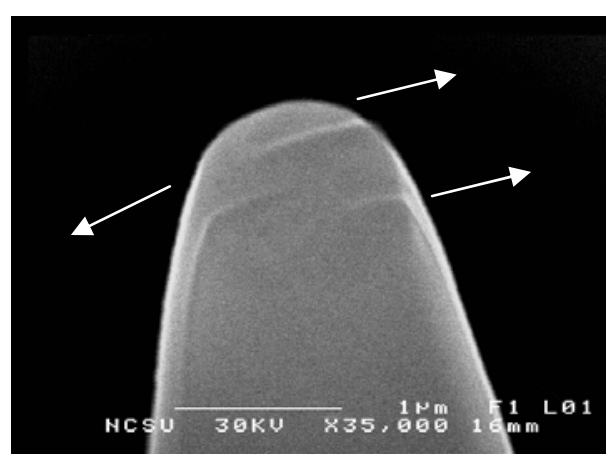

Figure 4: 3 linescans were performed off of the pillar to induce growth of rods. The linescans were performed perpendicular to the pillar's tip. The linegrowth followed the pillar down the side. instead. of growing off the top laterally. Scan directions are indicated. 